\title{
AlP inactivation leads to pituitary enlargement in the zebrafish embryo model
}

\author{
Marko Stojanovic ${ }^{1}$, Elena Daniela Aflorei², Imelda McGonnell ${ }^{3}$, Márta Korbonits ${ }^{2}$
}

${ }^{1}$ Neuroendocrine Unit, Clinic for Endocrinology, Diabetes and Metabolic Diseases,

Clinical Center of Serbia, Belgrade, Serbia, School of Medicine, University of Belgrade, Serbia

${ }^{2}$ Department of Endocrinology, William Harvey Research Institute, Queen Mary University of London, UK

${ }^{3}$ Department of Comparative Biomedical Sciences, Royal Veterinary College, University of London, UK e-mail: markostoj@yahoo.com

CONCLUSION: AIP Morpholino Knock Down zebrafish embryos demonstrate brain, pericardium, eye, and swim bladder anomalies along with general developmental delay, pointing to wide developmental role of AIP gene. AIP Morphant embryos exhibit larger surface of PRL immunostaining in the pituitary compared to controls suggesting possible increase in proliferative activity (hyperplasia or tumour) at the pituitary level in the absence of AIP gene function.

INTRODUCTION: Patients with aryl hydrocarbon receptorinteracting protein (AIP) gene mutations are predisposed to development of large, invasive, $\mathrm{GH}$ - or PRL-secreting pituitary tumours that typically occur at a younger age and are often resistant to medical treatment. The zebrafish (ZF) model represents close anatomical and functional similarities to the human neuroendocrine system.

MATERIAL and METHODS: AIP knock down (KD) ZF embryos were generated using antisense morpholino oligonucleotides injected at the one-cell stage. (Fig 1) Control embryos were injected with 5-base mispaired oligonucleotide as control morpholinos (CM), and wild type (WT) embryos from the same batch were used as uninjected controls. All embryos were incubated in the same conditions for 5 days, and observed during development. At 120 hours post fertilization (hpf) whole mount immunostaining of $K D, C M$ and WT ZF embryos was performed with anti-PRL antibodies (rabbit anti-salmon polyclonal 1:2000, Dr Takahashi, Japan) and then a total of 15 embryos ( 5 from each group) were randomly selected for digital microscopy. Image analysis software (NIH ImageJ 1.48v) was employed to assess pituitary staining

Figure 1a. Adult Danio rerio - Tupfel Long Fin Fig 1b. Microinjection in one-cell stage embryos

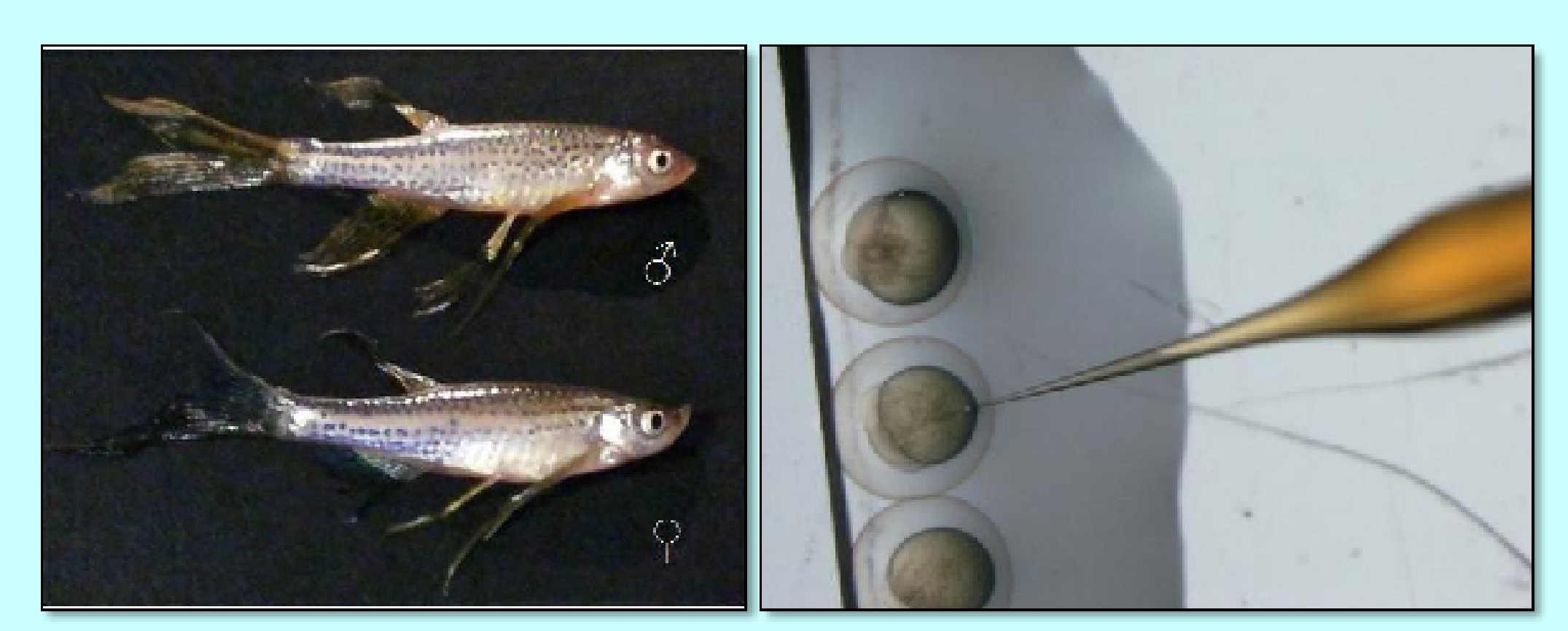

RESULTS: Overall developmental delay and retardation was observed in the AIP KD ZF compared to both control groups (WT and CM). (Fig 2a) KD embryos exhibited reduced total body length (Fig 2b), transitory midbrain enlargement (Fig 3), pericardium enlargement (Fig 4) and swim bladder under-development (Fig 2a). The shape, size and position of the pituitary were assessed by PRL staining. Pituitary in the AIP morphants appeared to be larger, shifted more ventrally and had a round shape compared to the oval or kidney shaped pituitary in the WT (Fig. 5a). Pituitary size in AIP morphants $\left(1621.9 \pm 87.2 \mu \mathrm{m}^{2}\right)$ was significantly larger then in WT $\left(574.1 \pm 357.8 \mu \mathrm{m}^{2} \mathrm{p}=0.04\right)$ and CM $\left(626.0 \pm 223.6 \mu \mathrm{m}^{2}\right.$ $\mathrm{p}=0.02$ ) with no statistical difference between the two control groups $(p=0.90)$. (Fig. $5 b)$

Figure 2a AIP Morhpant zebrafish (ZF) phenotype compared to control morpholinos. (a: midbrain, b: ericardium, c: swim bladder) Fig $2 b-$ Reduced average body length in AIP Morphant ZF $\left({ }^{*} p<0.05{ }^{* *} p<0.01\right)$
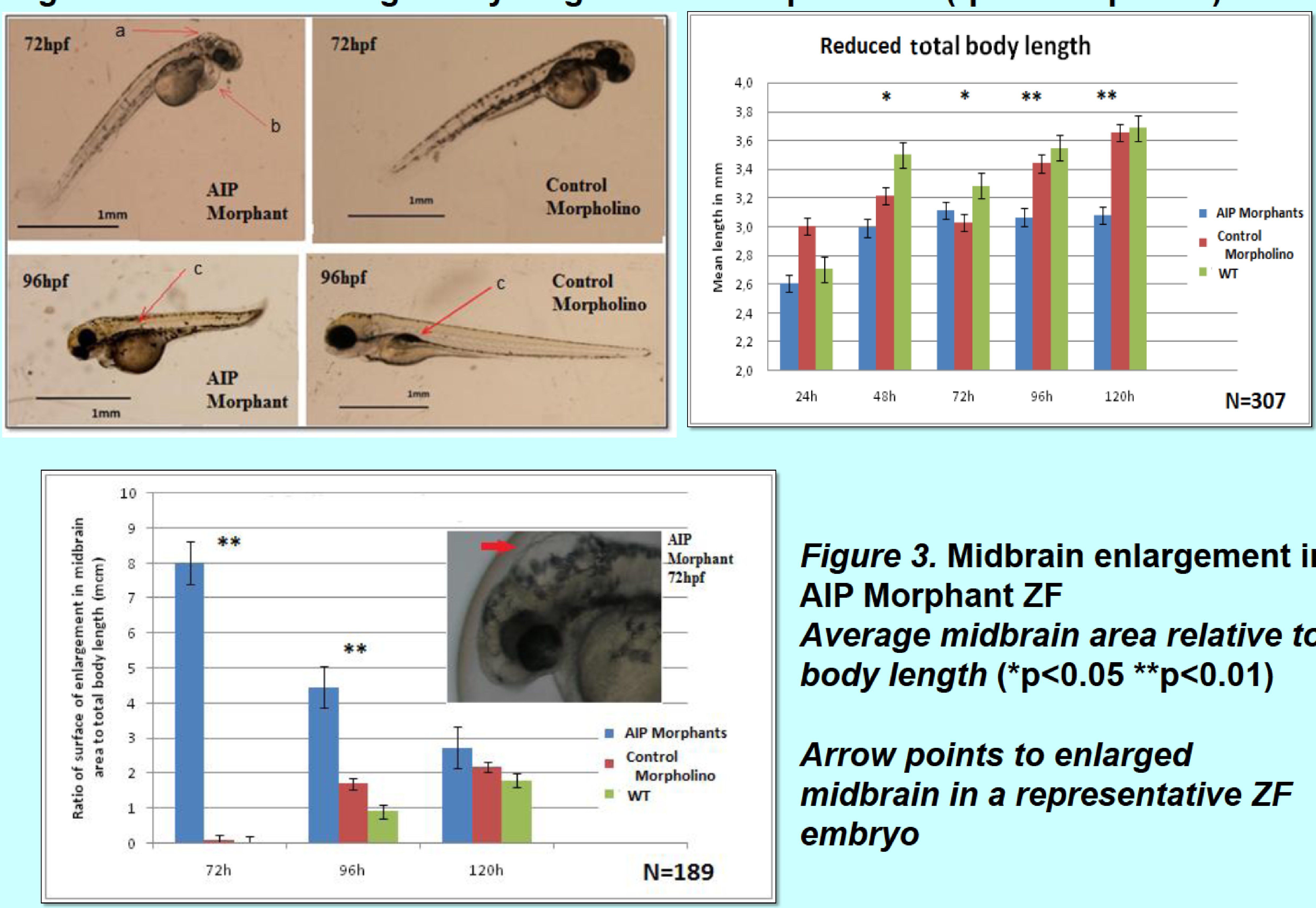

Figure 3. Midbrain enlargement in AIP Morphant ZF

Average midbrain area relative to body length $\left({ }^{*} p<0.05{ }^{* *} p<0.01\right)$

Arrow points to enlarged midbrain in a representative $\mathrm{ZF}$ embryo

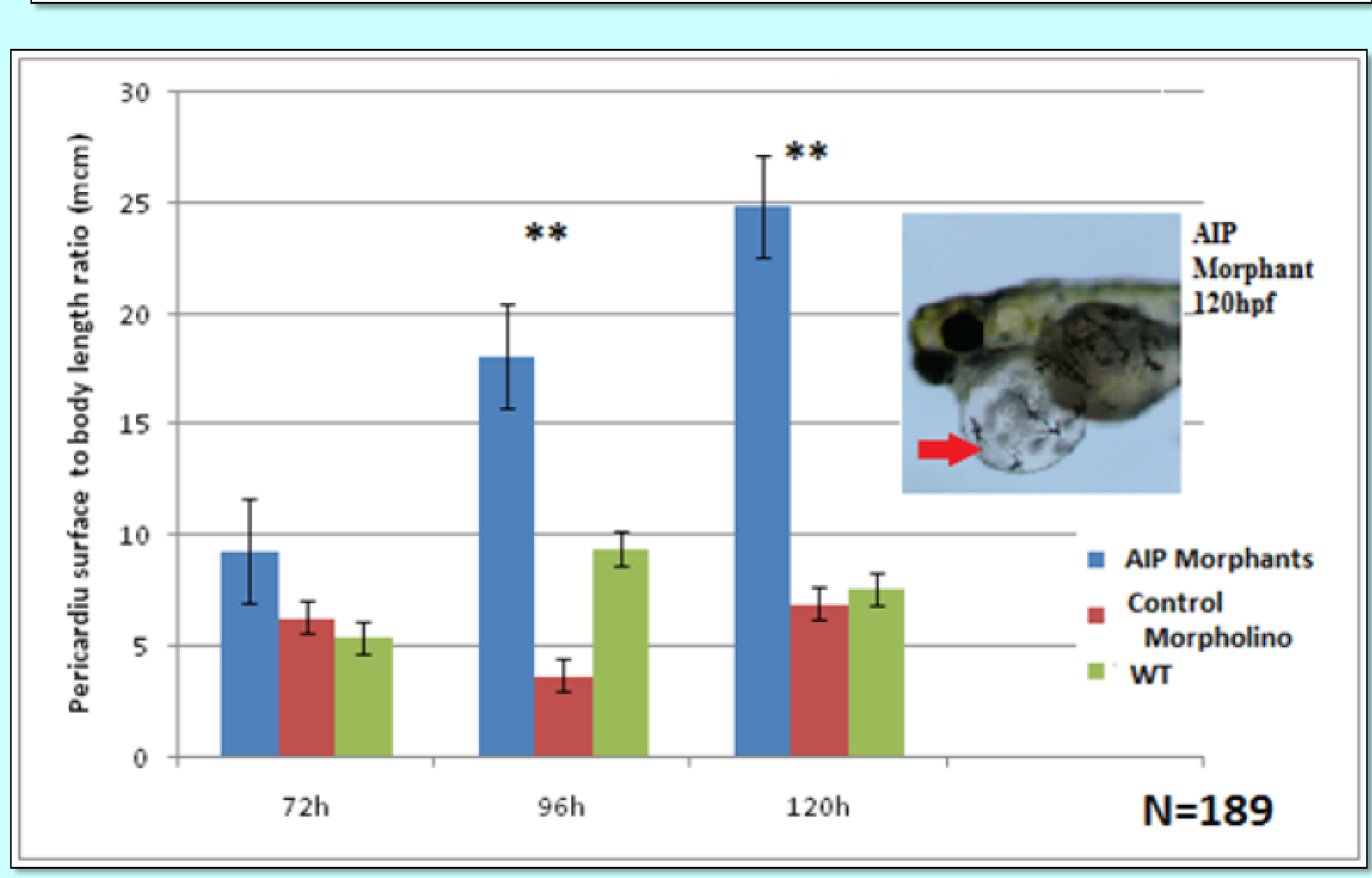

Figure 4: Pericardium enlargement in AIP Morphant ZF Average pericadium area relative to body length $\left({ }^{*} p<0.05{ }^{* *} p<0.01\right)$

Arrow points to enlarged pericardium in a representative ZF embryo

Figure 5a: Whole mount Immunostaining Anti PRL - Arrows point to pituitary Figure 5b: Average pituitary size in AIP Morhant ZF assesed by PRL immunostaining area. $\left({ }^{*} p<0.05{ }^{* *} p<0.01\right)$

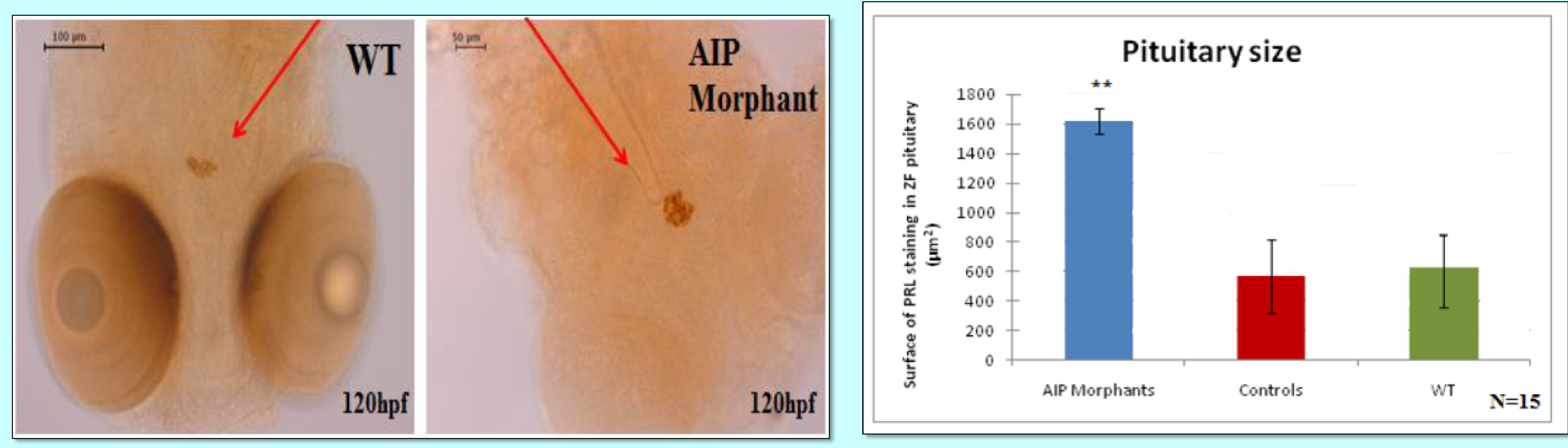

Supported by research grants from: 